\title{
Procalcitonina para el diagnóstico de la sepsis tardía en recién nacidos de muy bajo peso de nacimiento
}

\author{
Raúl Bustos B. y Heriberto Araneda C.
}

\section{Procalcitonin for the diagnosis of late onset sepsis in newborns of very low birth weight}

Procalcitonin (PCT), a prohormone of calcitonin has been described as a specific biomarker of sepsis. Objectives: To compare the predictive value of PCT, C reactive protein (CRP) and white blood cell count (WBC) for the diagnosis of late onset sepsis (LOS) in very low birth weight (VLBW) neonates. Methods: We prospectively determined the serum concentration of PCT, CRP and WBC in 53 VLBW newborns with clinical suspicion of LOS. 25 had confirmed sepsis with positive blood culture; 28 had clinical sepsis (negative blood cultures) Results: PCT levels were significantly higher in the infected group $(3.0 \mathrm{ng} / \mathrm{ml})$ compared to the non infected group $(0.4 \mathrm{ng} / \mathrm{ml})$ $(\mathrm{p}<0.05)$. PCT had the highest area under the ROC curve $0.83(95 \%$ CI 0.70-0.92) $\mathrm{p}=0.001$ compared to CRP $0.51(95 \%$ CI $0.37-0.65)$ and WBC 0.53 (95\%CI 0.38-0.66) for the diagnosis of LOS . The best PCT cut off value was $0.9 \mathrm{ng} / \mathrm{ml}$. The sensitivity, specificity and negative predictive value were $88 \%, 72 \%$ and $87 \%$, respectively. Conclusions: The determination of PCT could be more useful than CRP and WBC in the diagnosis of LOS in VLBW newborns.

Key words: Procalcitonin, sepsis, newborn, biomarkers.

Palabras clave: Procalcitonina, sepsis, recién nacidos, biomarcadores.

\section{Introducción}

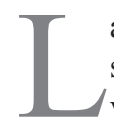

a sepsis neonatal tardía (SNT), definida como la sepsis que se presenta después de las 72 horas de vida, es una causa importante de morbilidad y mortalidad en los recién nacidos de muy bajo peso (RNMBP). De acuerdo a la red neonatal del National Institute of Child Health and Human Development de los Estados Unidos de América (E.U.A.), la incidencia de sepsis tardía en RNMBP es de $21 \%$, valor diez veces superior a la incidencia de sepsis precoz. Por otro lado, la mortalidad es tres veces mayor en aquellos RNMBP que presentan una SNT comparados a aquellos que evolucionan sin sepsis ${ }^{1}$. Este grupo de recién nacidos (RN) presenta un déficit en la inmunidad humoral y celular. Al nacer prematuramente, reciben menos inmunoglobulinas transplacentarias y además una función de complemento y fagocitosis deficiente en relación a los $\mathrm{RN}$ a término. Junto a lo anterior, el uso de catéteres centrales, la ventilación mecánica, estadías hospitalarias prolongadas y el empleo de algunos medicamentos como corticoesteroides, bloqueadores $\mathrm{H} 2$ y cefalosporinas, hacen de los RNMBP un grupo altamente susceptible a las infecciones. El diagnóstico de la sepsis neonatal es un desafío para el médico clínico, dada la naturaleza no específica de los signos y síntomas de la sepsis en RN prematuros. Como consecuencia de lo anterior, el sobreuso de antimicrobianos es una situación frecuente en las unidades de neonatología creando un ambiente para la emergencia de resistencia bacteriana y un potencial pronóstico desfavorable ${ }^{2}$. También, en la mayoría de los casos los resultados microbiológicos estarán disponibles a las 48-72 horas, por lo que la identificación precoz de los casos infectados es reconocida como uno de los mayores problemas en los RNMBP.

A pesar de que en la literatura científica aparecen propuestas de usar parámetros fisiológicos, índices hematológicos o perfiles de citoquinas al momento de la sospecha de la sepsis a fin de identificar con certeza aquellos RNMBP infectados, hoy en día no es posible realizar una recomendación de un test exclusivo para el diagnóstico de esta condición ${ }^{3-7}$.

La procalcitonina (PCT), ha sido descrita como un marcador específico de infecciones bacterianas. La PCT es un propéptido de la hormona calcitonina constituido por 116 aminoácidos, desprovisto de actividad hormonal y de producción extra-tiroidea, que se eleva rápidamente en pacientes con infecciones bacterianas. Esta respuesta se ha relacionado con la gravedad de la infección, ya que en infecciones graves puede alcanzar concentraciones entre 10 y $1.000 \mathrm{ng} / \mathrm{mL}$, niveles que decrecen rápidamente después de una terapia antimicrobiana adecuada. En individuos sanos, los valores séricos de PCT son casi indetectables $(0,033 \pm 0,003 \mathrm{ng} / \mathrm{ml})$ ascendiendo levemente en infecciones virales, trauma o cirugía. En voluntarios
Hospital Guillermo Grant Benavente, Concepción, Chile. Unidad de Cuidados Intensivos Pediátricos (RB). Universidad de Concepción, Concepción, Chile Laboratorio de Perinatología (HA).

Los autores no tienen conflictos de interés con el material presentado

Fuente de Financiamiento: Proyecto: No 203088025-10 Dirección de Investigación, Universidad de Concepción.

Recibido: 29 de febrero de 2012 Aceptado: 11 de septiembre de 2012

Correspondencia a: Raúl Bustos Betanzo robustos64@yahoo.com.an 
se ha observado que aparece en la sangre después de tres horas de la inyección de endotoxina, alcanzando concentraciones máximas a las seis horas, pudiendo permanecer elevada por 24 horas $^{8-10}$.

El nacimiento, la adaptación extrauterina y la colonización bacteriana intestinal estimulan una reacción de fase aguda con liberación de PCT, por lo que en RN normales las concentraciones de PCT llegan a valores pico a la 24 $\mathrm{h}$ de vida $(1,0-2,5 \mathrm{ng} / \mathrm{ml})$ alcanzando valores normales a las 72 horas $^{11-12}$.

En adultos críticamente enfermos, la PCT se ha utilizado en la diferenciación entre síndrome de respuesta inflamatoria sistémica de origen no infeccioso y la sepsis, para determinar la progresión del síndrome séptico, evaluar la respuesta al tratamiento y estimar pronóstico ${ }^{13-15}$. Una estrategia terapéutica basada en la medición de los niveles de PCT ha logrado reducir el sobreuso de antimicrobianos en infecciones respiratorias bajas y en pacientes sépticos en $\mathrm{UCI}^{16-17}$.

Por lo heterogéneo de los estudios de la PCT en RN, en términos del momento en su determinación, la población estudiada $-\mathrm{RN}$ a término o prematuros- y los valores óptimos de corte obtenidos, la utilidad y las ventajas de la PCT como biomarcador de sepsis en RN es aún objeto de debate.

El objetivo de la presente investigación fue comparar la utilidad de la $\mathrm{PCT}$ con la proteína $\mathrm{C}$ reactiva $(\mathrm{PCR}) \mathrm{y}$ el recuento de leucocitos (RL) como predictor de sepsis neonatal tardía en una cohorte de RNMBP.

\section{Pacientes y Métodos}

Estudio prospectivo, observacional, realizado en la Unidad de Neonatología del Hospital Guillermo Grant Benavente, Concepción, durante 18 meses. Este trabajo fue aprobado por el comité ético científico de nuestro hospital. Para ser elegibles en el estudio los RN debieron cumplir con los siguientes criterios: Edad gestacional menor a 32 semanas y/o peso de nacimiento menor a 1.500 gr, ser mayores a 72 horas de vida y no haber recibido antimicrobianos las 48 horas precedentes. Después de obtener el consentimiento informado, los recién nacidos elegibles fueron enrolados y monitoreados por signos clínicos de sepsis. Tres o más de los siguientes criterios fueron requeridos para iniciar una evaluación por sepsis: inestabilidad térmica definida como $\mathrm{T}^{\circ}<36,5 \mathrm{o}>37,5^{\circ} \mathrm{C}$ en dos tomas consecutivas; disfunción cardiovascular definida como taquicardia persistente $(>180 \mathrm{lpm})$, bradicardia $(<80 \mathrm{lpm})$, perfusión periférica disminuida, hipotensión arterial ( $<2 \mathrm{DS}$ para la edad gestacional); disfunción respiratoria manifestada como aumento del soporte ventilatorio, requerimientos de oxígeno, taquipnea $>$ a $70 \mathrm{rpm}$ o aparición de apneas; disfunción gastrointestinal definida como distensión abdominal asociada a vómitos aumento de residuo gástrico o deposiciones con sangre; anomalías en los exámenes bioquímicos de laboratorio acidosis metabólica, hiperglicemia; exámenes hematológicos anormales (leucocitosis $>15.000 / \mathrm{mm}^{3}$ o leucopenia $<5.000 / \mathrm{mm}^{3}$, trombocitopenia $<100.000 / \mathrm{mm}^{3}$ )

Los criterios de exclusión fueron presencia de malformaciones incompatibles con la vida y ausencia de consentimiento informado.

Definiciones: Sepsis tardía se definió como el aislamiento de microorganismos patógenos de uno o más sitios normalmente estériles obtenidos después de las 72 horas de vida. Para Staphylococcus coagulasa negativa la presencia de dos hemocultivos positivos o un hemocultivo positivo y signos clínicos de sepsis se consideraron como sepsis tardía.

Ensayos: Una vez que la infección fue sospechada se realizó toma de dos hemocultivos, RL, PCR y PCT. Los hemocultivos fueron procesados por el laboratorio de microbiología del Hospital Guillermo Grant Benavente usando el sistema BACTEC ${ }^{\circledR}$ (Becton Dickinson MD E.U.A.). Una vez obtenidas las muestras para medición de PCT, fueron centrifugadas a $1.500 \mathrm{rpm}$ por 15 minutos y el suero aliquotado fue almacenado a $-20^{\circ} \mathrm{C}$ hasta el momento del ensayo. La medición de PCT se realizó por ensayo inmunoluminométrico (LUMI test PCT Brahms Diagnostica Berlín Alemania) cuyo limite de detección se sitúa en 0,5 ng/mL. La medición de PCR se hizo por turbidimetria (Behring BN2 Marburg Alemania). El RL se realizó por el sistema Coulter STKS (Beckmann Coulter, E.U.A.)

Análisis estadístico: Los valores son expresados en mediana (rango). La comparación entre los grupos se realizó con el test de Mann-Whitney U. La eficacia diagnóstica de los diferentes marcadores para el diagnóstico de sepsis tardía se calculó a través de una curva ROC (receiver operating characteristic). Se calculó la sensibilidad especificidad y valores predictor positivo $\mathrm{y}$ negativo para cada marcador. Un valor $\mathrm{p}<\mathrm{a} 0.05$ fue considerado como estadísticamente significativo. El análisis estadístico se realizo con el programa SSPS 12 (SPSS Inc .Chicago, Ill, E.U.A.).

\section{Resultados}

Cincuenta y tres RNMBP con peso y la edad gestacional promedio de $980(580-1.570)$ y 30 semanas de edad gestacional (23-35 sem) fueron evaluados por posible sepsis tardía al día 9 (3-40) de vida. Veinticinco RNMBP presentaron sepsis con hemocultivo positivo, con una 
incidencia de $20,8 \%$ y hubo 28 sepsis clínica con hemocultivo negativo; no existieron diferencias estadísticamente significativas en las características demográficas y edad de inclusión al estudio entre ambos grupos (Tabla 1).

De los 25 recién nacidos infectados, $100 \%$ presentaron sepsis, $3(12,5 \%)$ meningitis y $1(4 \%)$ paciente neumonía. Diecinueve infecciones fueron causadas por microorganismos grampositivos; de éstos, fue más frecuente Staphylococcus aureus resistente a meticilina. Seis casos correspondieron a sepsis por bacilos gramnegativos (Tabla 2). No se aislaron hongos.

Siete $(13 \%)$ de los 53 pacientes fallecieron, todos pertenecientes al grupo de RNMBP con hemocultivos positivos. Seis fallecieron de falla multiorgánica y uno de hemorragia intracerebral masiva. Cuatro de los fallecidos presentaron una sepsis por bacilos gramnegativos (Pseudomonas aeuruginosa 2, Klebsiella pneumoniae 1, Enterobacter cloacae 1).

Los niveles plasmáticos de PCT fueron significativamente más elevados $(p=0,003)$ en los recién nacidos con sepsis confirmada $(3,0 \mathrm{ng} / \mathrm{ml})$ comparados a aquellos recién nacidos con sepsis clínica $(0,4 \mathrm{ng} / \mathrm{ml})$. No existió diferencia estadísticamente significativa en los valores de la PCR y en el RL entre ambos grupos (Tabla 3).

Al categorizar los pacientes según el microorganismo aislado en el hemocultivo, observamos que los niveles de PCT fueron significativamente más elevados $(p=0,001)$ en aquellos RNMBP con sepsis secundaria a infección por bacilos gramnegativos $(73,0 \mathrm{ng} / \mathrm{ml})$ en comparación a infecciones por grampositivos $(2,0 \mathrm{ng} / \mathrm{ml})$. Las concentraciones de la PCR y RL no arrojaron diferencias significativas. Los niveles de PCT y de la PCR fueron más elevados en los RNMBP fallecidos al compararlos con los sobrevivientes 15,0 vs 1,0 ng $/ \mathrm{ml},(\mathrm{p}=0,006)$ y 113,5 vs $47 \mathrm{mg} / \mathrm{L}(\mathrm{p}=0,017)$, respectivamente.

Se evaluó la eficacia diagnóstica de la PCT, PCR y RL para el diagnóstico de sepsis mediante la curva ROC. La PCT presentó el área más alta bajo la curva (IC 95\%) comparada a los otros marcadores: PCT 0,83 $(0,70-0,92)$ $\mathrm{p}=0,0001 v s$ PCR $0,51(0,37-0,65) \mathrm{p}=0,9$ vs RL 0,53 $(0,38-0,66) \mathrm{p}=0,73$ (Figuras 1-3).

Los puntos de corte óptimos obtenidos a partir de la curva ROC, para la PCT fueron $0,9 \mathrm{ng} / \mathrm{ml}$ con una sensibilidad de $88 \%$ especificidad $71,4 \%$ y un valor predictor negativo de $87 \%$. El LH ratio positivo fue de 3,0 y el LHR negativo fue de 0,17 (Tabla 4).

\section{Discusión}

En este estudio, la medición de PCT aparece como un marcador superior a la PCR y el RL en el diagnóstico de sepsis tardía en RNMBP cuando se utiliza el hemocultivo como "estándar de oro" de infección.

\begin{tabular}{|lccc|}
\hline \multicolumn{4}{|c|}{ Tabla 1. Características de los RNMBP incluidos en el estudio } \\
\hline \\
\begin{tabular}{|lccc} 
Sepsis clínica \\
(n: $\mathbf{2 8})$
\end{tabular} & $\begin{array}{c}\text { Sepsis confirmada } \\
\text { (n: } \mathbf{2 5})\end{array}$ & P \\
Peso de nacimiento (g) & $980(590-1.570)$ & $980(580-1.460)$ & 0,92 \\
Edad gestacional (sem) & $28,5(24-33)$ & $30,0(23-35)$ & 0,57 \\
Sexo M/F & $12 / 16$ & $12 / 13$ & 0,53 \\
Edad de inclusión en el estudio (días) & $8,5(3-32)$ & $10(3-40)$ & 0,26 \\
\hline RNMBP: recién nacidos de muy bajo peso & & \\
\hline
\end{tabular}

\begin{tabular}{|lc|}
\multicolumn{2}{|c|}{ Tabla 2. Microorganismos aislados de sangre } \\
en 25 recién nacidos & n \\
\hline Grampositivos & 7 \\
Staphylococcus coagulasa negativa & 6 \\
Staphylococcus aureus RM & 5 \\
Staphylococcus aureus SM & 1 \\
Listeria monocytogenes & \\
Bacilos gramnegativos & 4 \\
Klebsiella pneumoniae & 1 \\
Enterobacter sp & 1 \\
Pseudomonas aeruginosa & 25 \\
\hline Total & \\
\hline RM: resistente a meticilina. SM: sensible a meticilina. & \\
\hline
\end{tabular}

Tabla 3. Valores de procalcitonina (PCT), proteína C reactiva (PCR) y recuento de leucocitos (RL) Valores expresados en mediana (rango)

\begin{tabular}{lccc|} 
& Sepsis clínica & Sepsis confirmada & P \\
PCT ng/ml & $0,4(0,1-125)$ & $3,0(0,3-208)$ & 0,0031 \\
\hline PCR mg /l & $48,5(1-111)$ & $49,0(0-142)$ & 0,89 \\
Recuento de leucocitos (céls/mm $\left.{ }^{3}\right)$ & $8.800(3.000-25.900)$ & $12.000(1.800-22.800)$ & 0,73 \\
\hline
\end{tabular}

Tabla 4. Precisión diagnóstica de la procalcitonina (valor de corte $0,9 \mathrm{ng} / \mathrm{ml}$ ), proteína C reactiva (valor de corte $111 \mathrm{mg} / \mathrm{L}$ ) y recuento de leucocitos (valor de corte $11,2 \times 103)$. Resultados expresados como porcentaje ( $95 \%$ IC)

\begin{tabular}{|lccc|} 
& Procalcitonina & Proteína C reactiva & Recuento de leucocitos \\
Sensibilidad \% & $88(69-97)$ & $12(3-31)$ & $52(31-72)$ \\
Especificidad \% & $72(51-87)$ & $100(88-100)$ & $64(44-81)$ \\
LR positivo & 3 & - & 1,4 \\
LR negativo & 0,2 & 0,9 & 0,8 \\
VPP \% & 73 & 100 & 56 \\
VPN \% & 87 & 56 & 60 \\
\hline LR: likelihood ratio. VPP: Valor predictor positivo. VPN: Valor predictor negativo. \\
\hline \multicolumn{4}{l}{} \\
\hline
\end{tabular}


Figura 1. Curva ROC de la PCT para el diagnóstico de sepsis. AUC 0,83 (IC95\% 0,76-0,92) $\mathrm{p}=0,0001$

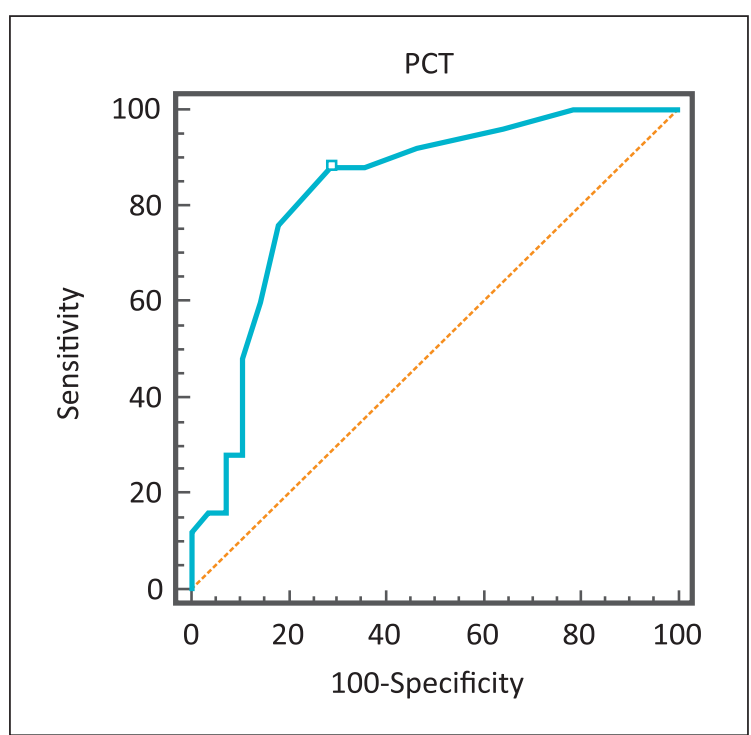

Figura 3. Curva ROC del RL para el diagnóstico de sepsis AUC 0,53 95\% IC 0,38-0,66) $p=0,73$.

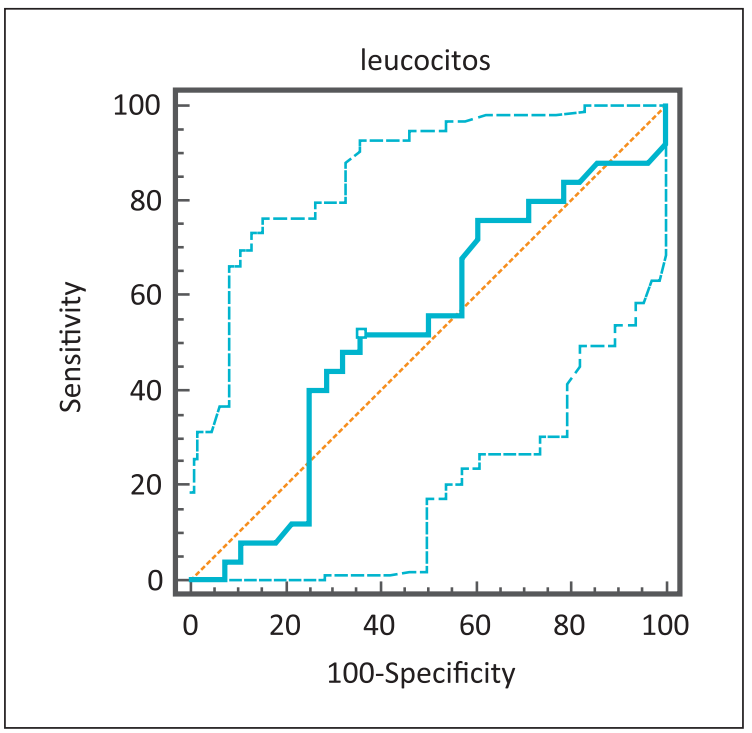

Nuestros hallazgos son concordantes con Vazzalar, que en 67 RNMBP estableció que los niveles de PCT fueron significativamente superiores en los pacientes infectados $(5,47 \mathrm{ng} / \mathrm{ml})$ en relación a los no infectados $(0,43 \mathrm{ng} /$ $\mathrm{ml})$. Con un valor de corte de $0,5 \mathrm{ng} / \mathrm{ml}$ la PCT obtiene una sensibilidad de $97 \%$ en relación al $73 \%$ de la $\mathrm{PCR}^{18}$. Jacquot reportó un valor de corte de la PCT en $0,6 \mathrm{ng} / \mathrm{ml}$ en 73 RNMBP siendo el valor predictor para diagnóstico de sepsis tardía de la PCT superior a la PCR (área bajo la curva ROC 0,88 vs 0,77) alcanzando una sensibilidad de $100 \%$ y un valor predictor negativo de $100 \%$. Los autores concluyen que un valor inferior a este punto de corte

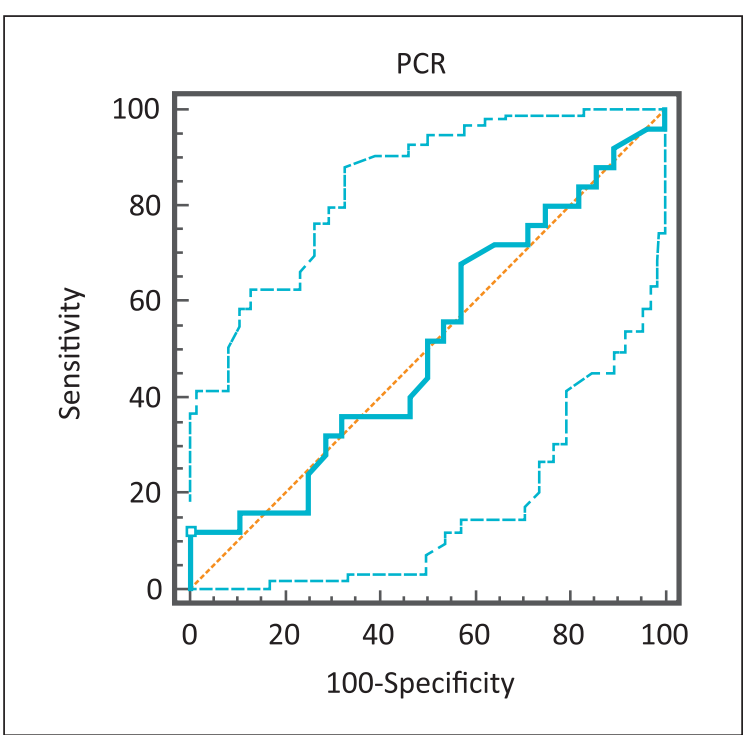

Figura 2. Curva ROC de la PCR para el diagnóstico de sepsis AUC $0,51(95 \%$ IC $0,36-0,65) p=0,90$.

podría excluir una sepsis tardía. Finalmente, un estudio multicentrico italiano demostró que un nivel plasmático de PCT $>2,4 \mathrm{ng} / \mathrm{ml}$ alcanza una sensibilidad de $65 \%$ y especificidad de $84 \%$ para el diagnostico de sepsis tardía en RNMBP ${ }^{19-20}$.

Como nuestra observación, otros autores han descrito concentraciones de PCT superiores en RNMBP infectados por microorganismos gramnegativos al compararlos con infecciones por $S$. coagulasa negativa $(7,6 \mathrm{vs} 0,76 \mathrm{ng} /$ $\mathrm{ml}$ y 9,36 vs $2,69 \mathrm{ng} / \mathrm{mL}$ ), respectivamente. Lo anterior podría ser explicado por estímulo del lipopolisacarido de las bacterias gramnegativas asociado al compromiso multisistémico que acompaña este tipo de infecciones ${ }^{21-23}$.

El valor de corte ideal para la PCT es objeto de discusión. En pacientes pediátricos, el umbral para el diagnóstico de infecciones bacterianas se ha estimado entre 0,5 y $2,0 \mathrm{ng} / \mathrm{ml}$, dependiendo del estudio y del tipo de pacientes reclutados (sala de emergencia o unidad de cuidados intensivos). En $\mathrm{RN}$ a término y pre-término se han reportados valores de corte de 0,6 a $2,3 \mathrm{ng} / \mathrm{ml}$ con sensibilidades que van desde de 97 a $69 \%{ }^{24}$.

Una fortaleza de nuestro estudio fue que la medición de la PCT, realizada después de las 72 h de vida, permitió obviar el aumento fisiológico de los niveles de PCT que se producen las primeras $48 \mathrm{~h}$ en $\mathrm{RN}$ normales, $\mathrm{y}$, a diferencia de otros reportes en la literatura médica, estudiamos un grupo homogéneo de RNMBP con sospecha clínica de sepsis nosocomial. En este sentido, una revisión sistemática y meta-análisis reciente de 16 estudios que incluyeron 1.959 RN, ha demostrado que el uso de la PCT en RN es más útil en el diagnóstico de la sepsis tardía con un área bajo la curva de 0,95 comparada a $\mathrm{RN}$ con sepsis antes 
de las $72 \mathrm{~h}$ de vida, que presentó un área bajo la curva de $0,78^{25}$.

Las quimioquinas y citoquinas han sido extensivamente estudiadas en la década pasada como biomarcadores de sepsis en RN. De esta importante categoría de mediadores, la citoquina pro-inflamatoria IL-6, la citoquina anti-inflamatoria IL-10 y la quimioquina IL-8 serían de potencial utilidad, incluso superior a Ia PCT, según algunos estudios, para el diagnóstico precoz de la sepsis y para predecir la gravedad de la infección al momento de la presentación de la sepsis ${ }^{26-27}$.

A pesar de sus propiedades favorables, el uso de estos mediadores no ha sido integrado a la práctica clínica diaria. Sus costos elevados, una metodología no automatizada y laboriosa para su medición, han sido los mayores obstáculos que han impedido la evolución de las citoquinas como test diagnósticos de rutina de infección en las unidades de neonatología ${ }^{28}$.

Algunas limitaciones de nuestro trabajo la constituyen el escaso número de pacientes incluidos y su carácter unicéntrico. Al utilizar como "estándar de oro" de infección el hemocultivo, es posible que algunos casos de verdadera infección del torrente sanguíneo no hayan sido confirmados por cultivos sanguíneos falsamente negativos debido a factores como el uso de antimicrobianos intraparto, extracción de volúmenes pequeños de sangre, lugar inadecuado para la extracción. Finalmente, el ensayo para la medición de PCT que se utilizó en este estudio y en la mayoría de las experiencias publicadas, es un ensayo inmunoluminométrico manual cuyo nivel de sensibilidad reportado es de $0,5 \mathrm{ng} / \mathrm{ml}$, considerando que este valor excede 10 veces los valores normales descritos para la PCT. Con este ensayo, aumentos moderados de la PCT pueden no ser detectados, de tal modo que pacientes con bacteriemias podrían tener niveles de PCT en el rango indeterminado. Al momento de realizar este estudio no existía otro método disponible de medición de la PCT en nuestro país.

La aparición reciente de ensayos para la PCT auto- matizados y ultrasensibles -sensibilidad de $0,06 \mathrm{ng} / \mathrm{ml}-$ (Kryptor ${ }^{\circledR}$ BRAHMS o VIDAS ${ }^{\circledR}$-bioMérieux) permitiría mejorar el diagnostico de este grupo de pacientes con niveles indeterminados de PCT y validar un valor de corte que ayude a reducir el uso innecesario de antimicrobianos en la UCI neonatal ${ }^{29-30}$.

\section{Conclusiones}

Nuestros hallazgos sugieren que la medición de niveles de la PCT al momento de la presentación clínica, es más útil que la PCR y el RL en el diagnóstico de sepsis tardía en RNMBP.

\section{Resumen}

La procalcitonina (PCT) ha sido descrita como un marcador especifico de infecciones bacterianas. Objetivos: Comparar el valor predictor de la PCT, Proteína $C$ reactiva (PCR) y recuento de leucocitos $(R L)$ en el diagnostico de sepsis tardía en recién nacidos de muy bajo peso (RNMBP). Métodos: Estudio prospectivo. Niveles de PCT, PCR y RL fueron determinados en 53 RNMBP con sospecha clínica de sepsis. 25 presentaron sepsis confirmada por hemocultivo, 28 sepsis clínica (hemocultivo negativo). Resultados: Los niveles de PCT fueron significativamente mas elevados $(\mathrm{p}<0,05)$ en los RNMBP con sepsis confirmada $(3,0 \mathrm{ng} / \mathrm{ml})$ comparados a aquellos con sepsis clínica $(0,4 \mathrm{ng} / \mathrm{ml})$ La PCT presentó el área mas alta bajo la curva ROC 0,83 (95\%IC 0,7-0,92) $(\mathrm{p}=0,0001)$ comparada a la PCR 0,51(95\%IC 0,37-0,65) y el RL 0,53 (95\%IC 0,38-0,66) para el diagnóstico de sepsis. Un valor de corte de PCT de $0,9 \mathrm{ng} / \mathrm{ml}$ presentó una sensibilidad y especificidad de 88 y $72 \%$ respectivamente para el diagnóstico de sepsis, con un valor predictor negativo de 87\%. Conclusiones: La medición de la PCT podria ser un biomarcador de mayor utilidad que la PCR y el RL en el diagnóstico de la sepsis tardía en RNMBP.

\section{Referencias bibliográficas}

1.- Stoll B J, Hansen N, Fanaroff A A, Wright L L, Carlo W A, Ehrenkranz R A, et al. Late onset sepsis in very low birth weight neonates: The experience of the NHICD Neonatal Research Network. Pediatrics 2002; 110: 285-91.

2.- Clark R H, Bloom B T, Spitzer A R, Gerstmann D R. Empiric use of ampicillin and cefotaxime, compared with ampicillin and gentamicin, for neonates at risk for sepsis is associated with an increased risk of neonatal death. Pediatrics 2006; 117: 67-74.
3.- Griffin M P, Lake D E, Bissonette E A, Harrell F E Jr, O'Shea T M, Moorman J R. Heart rate characteristics: novel physiomarkers to predict neonatal infection and death. Pediatrics 2005; 116: 1070-4.

4.- Dalgic N, Ergenekon E, Koc E, Atalay Y. NOSEP and clinical scores for nosocomial sepsis in a neonatal intensive care unit. J Trop Pediatr 2006; 52: 226-7.

5.- Smulian J C, Bhandari V, Campbell W A, Rodis J F, Vintzileos A M. Value of umbilical artery and vein levels of interleukin- 6 and soluble intracellular adhesion molecule- 1 as predictors of neonatal hematologic indices and suspected early sepsis. J Matern Fetal Med 1997; 6: 254-9.

6.- Okascharoen C, Sirinavin S, Thakkinstian A, Kitayaporn D, Supapanachart S. A bedside prediction-scoring model for late onset neonatal sepsis. J Perinatol 2005; 25: 778-83.

7.- Malik A, Hui C P, Pennie A R, Kirpalani H. Beyond the complete blood cell count and $\mathrm{C}$ reactive protein. A systematic review of modern diagnostic test for neonatal sepsis. Arch Pediatr Adolesc Med 2003; 157: 511-16.

8.- Grendel D, Bohuon C. Procalcitonin as a marker of bacterial infection Pediatr Infect Dis J 2000; 19: 679-88. 
9.- Assicot M, Grendel D, Carsin H, Raymond J, Guilbaud J, Bohuon C, et al. High serum procalcitonin concentrations in patients with sepsis and infections. Lancet 1993; 341: 515-18.

10.- Snider R H Jr, Nylen E S, Becker K L. Procalcitonin and its component peptides in systemic inflammation: Immunochemical characterization. J Investig Med 1997; 45: 552-60.

11.- Marchini G, Berggren V, Djilalai-Merzoug R, Hansson L O. The birth process initiates an acute phase reaction in the fetus-newborn infant Acta Pediatr 2000; 89: 1082-6.

12.- Chiesa C, Panero A, Rossi N, Stegagno M, De Giusti M, Osborn J F, et al. Serum procalcitonin concentrations in term delivering. Reliabilty of procalcitonin concentrations for the diagnosis of sepsis in critically ill neonates. Clin Infect Dis 1998; 26: 664-72.

13.- Harbarth S, Holeckova K, Froidevaux C, Pittet D, Ricou B, Grau GE, et al; Geneva Sepsis Network. Diagnostic value of procalcitonin, interleukin-6, and interleukin-8 in critically ill patients admitted with suspected sepsis Am J Respir Crit Care Med 2001; 164: 396-402.

14.- Clec'h C C, Ferriere F, Karoubi P Fosse J P, Cupa M, Hoang P, et al. Diagnostic and prognostic value of procalcitonin in patients with septic shock Crit Care Med 2004; 32: 1166-9.

15.- Jensen J U, Heslet L, Jensen T H, Espersen K, Steffensen $\mathrm{H}$, Tvede M. Procalcitonin increase in early identification of critically ill patients at high risk of mortality. Crit Care Med 2006 ; 34 : 2596-602

16.- Christ-Crain M, Stolz D, Bingisser R,
Müller C, Miedinger D, Huber P R, et al. Procalcitonin guidance of antibiotic therapy in community-acquired pneumonia: A randomized trial. Am J Respir Crit Care Med 2006; 174: 74-93.

17.- Nobre V, Harbarth S, Graf J D, Rohner P, Pugin J. Use of procalcitonin to shorten antibiotic treatment duration in septic patients: a randomized trial. Am J Respir Crit Care Med 2008; 177: 498-505.

18.- Vazzalwar R, Pina-Rodrigues E, Puppala L B, Angst D B, Schweig L. Procalcitonin as a screening test for late onset sepsis in preterm very low birth weight infants. J Perinatol 2005; 25: 397-402.

19.- Jacquot A, Labaune J M, Baum T H, Putet G, Picaud J Q Rapid quantitative procalcitonin measurement to diagnose nosocomial infections in newborn infants. Arch Dis Child Fetal Neonatal Ed 2009; 94: F345-8.

20.- Auriti C, Fiscarelli E, Ronchetti M P, Argentieri M, Marrocco G, Quondamcarlo A, et al. Procalcitonin in detecting neonatal hosocomial sepsis. Arch Dis Child Fetal Neonatal Ed 2012; 97 (5): F368-70.

21.- Fendler W M, Piotrowski A J. Procalcitonin in the early diagnosis of nosocomial sepsis in preterm neonates J Paediatr Child Health 2008; 44: 114-8.

22.- Kordek A. Concentrations of procalcitonin and C-reactive protein, white blood cell count, and the immature-to-total neutrophil ratio in the blood of neonates with nosocomial infections: Gram-negative bacilli vs coagulase-negative staphylococci. Eur J Clin Microbiol Infect Dis. 2011; 30: 455-7.

23.- López Sastre J B, Pérez Solís D, Roques
Serradilla V, Fernández Colomer B, Coto Cotallo G, Krauel Vidal X, et al. Procalcitonin is not sufficiently reliable to be the sole marker of neonatal sepsis of nosocomial origin. BMC Pediatr 2006; 6 (1): 16.

24. Van Rosum A M C, Wulkan R W, OudesluysMurphy A M. Procalcitonin as an early marker of infection in neonates and children. Lancet Infect Dis 2004: 620-30.

25.- Vouloumanou E K, Plessa E, Karageorgopoulos D E, Mantadakis E, Falagas E M. Serum procalcitonin as a diagnostic marker for neonatal sepsis: a systematic review and meta-analysis. Intensive Care Med 2011; 37: 747-62.

26.- Ng P C, Li K, Wong R P, Chui K M, Wong E, Li G, et al. Proinflammatory and antiinflammatory cytokine responses in preterm infants with systemic infections. Arch Dis Child Fetal Neonatal Ed 2003; 88: 209-13.

27.- Zeitoun A A, Gad S S, Attia F M, Abu Maziad A S, Bell E F. Evaluation of neutrophilic CD64, interleukin 10 and procalcitonin as diagnostic markers of early- and late-onset neonatal sepsis. Scand J Infect Dis 2010; 42: 299-305.

28.- Ng P C, Lam H S. Biomarkers for late-onset neonatal sepsis: cytokines and beyond. Clin Perinatol 2010; 37: 599-610.

29.- Becker K L, Snider R, Nylen E S. Procalcitonin assay in systemic inflammation, infection, and sepsis: Clinical utility and limitations Crit Care Med 2008; 36: 941-52.

30.- Schuetz P, Christ-Crain M, Muller B. Biomarkers to improve diagnostic and prognostic accuracy in systemic infections. Curr Opin Crit Care 2007; 13: 578-85. 